Meta

Journal des traducteurs

Translators' Journal

\title{
Bilan d'une décennie
}

\section{Daniel Eyraud}

Volume 19, numéro 1, mars 1974

La traduction médicale

URI : https://id.erudit.org/iderudit/002713ar

DOI : https://doi.org/10.7202/002713ar

Aller au sommaire du numéro

Éditeur(s)

Les Presses de l'Université de Montréal

ISSN

0026-0452 (imprimé)

1492-1421 (numérique)

Découvrir la revue

Citer cet article

Eyraud, D. (1974). Bilan d'une décennie. Meta, 19(1), 13-27.

https://doi.org/10.7202/002713ar

Ce document est protégé par la loi sur le droit d'auteur. L'utilisation des services d'Érudit (y compris la reproduction) est assujettie à sa politique d'utilisation que vous pouvez consulter en ligne.

https://apropos.erudit.org/fr/usagers/politique-dutilisation/
Cet article est diffusé et préservé par Érudit.

Érudit est un consortium interuniversitaire sans but lucratif composé de l’Université de Montréal, l'Université Laval et l'Université du Québec à Montréal. Il a pour mission la promotion et la valorisation de la recherche. https://www.erudit.org/fr/ 


\section{Bilan d'une décennie}

"Il n'est pas sûr qu'au-delà d'un certain seuil les emprunts massifs ne compromettent la spécificité culturelle d'une langue.»

RoBert DUBUC

a Le français n'est ni moins plastique ni moins ingénieux que l'anglais ; seulement cela dépend de ceux qui s'en servent.

AURÉLIEN SAUVAGEOT

" Il ne suffit pas de parler la même langue, il faut parler le même langage. *

Dr Alexandre Manurla

Il n'est rien de tel pour évaluer l'état de santé de la langue, dans un secteur donné de la science, que de remonter dans son passé. Pour ce qui nous regarde un recul de dix ans suffira.

En effet, à ce stade de son histoire, l'expression médicale en France semblait avoir atteint son étiage, sous l'effet d'une dégradation ambivalente, conjugant des facteurs apparemment contraires : la négligence et la gloriole, dont la résultante était l'opacité.

D'un côté un français parlé. Non le parler spontané et fleuri du peuple, mais un français gauche, empêtré, semé d'impropriétés, encombré de répétitions, farci de clichés et de formules préfabriqués à la mode du jour.

À l'opposé, un maniérisme « incroyable » où le soin de l'auteur était moins d'instruire que d'éblouir : phraséologie alambiquée, réhaussée de mots miroitants, prélevés sur le jargon de nos spécialistes ou sur la plus fraîche marée atlantique; bricolés avec des éléments grecs prétentieusement et souvent mal juxtaposés; voire jaillis du prurit d'écrire qui dévore la fleur de la génération montante.

$\mathrm{Ce}$ salmigondis plastronnait particulièrement dans certains éditoriaux professionnels, où il tendait à faire école. Comme par une sorte de parti pris, vocables de spécialistes, anglicismes, hellénismes, néologismes, accidentaient le discours, faisant trébucher ou immobilisant le lecteur. L'enseignant tournait le dos au vulgarisateur, c'est-à-dire à celui qui possède ensemble la maîtrise de son savoir et de sa langue maternelle.

Or, à qui s'adressent nos revues professionnelles? Qui en constitue 1a clientèle ? Qui, en somme, justifie et assure leur existence, sinon la masse des praticiens, les tâcherons du grand métier, les dépanneurs de la maladie, les besogneux de la base, sans lesquels il n'y aurait pas plus de sommités médicales qu'il n'y a jamais eu de généraux sans piétaille. Que signifiait cette cuistrerie à masque savan- 
tasse, qui trahissait jusqu'à la raison d'être du langage : assurer la communication entre les hommes?

Dans cette parade mirobolante resplendissaient d'un éclat non pareil les anglicismes.

Compétence et place me faisant défaut pour instruire le procès du langage médical actuel, je me bornerai à celui de l'anglomanic. Je dis bien l'anglomanie et non la langue anglaise, afin d'éliminer d'emblée la pièce maîtresse de la partie adverse : l'accusation de chauvinisme.

\section{PROMENADE DANS LES CÉVENNES}

Que l'on me permette une digression qui m'est personnelle et que je souhaite toutefois non haïssable.

J'ai exercé la médecine rurale aux confins du Velay et du Vivarais, dans un décor volcanique que domine le roi des Cévennes, le Mézenc, et qu'avoisine le pain de sucre du Gerbier, au pied duquel une auge rustique reçoit la première goulée de la Loire.

Là, se partagent les eaux entre Atlantique et Méditerranée; les influences religieuses entre papistes et parpaillots; les deux grands fonds dialectiques de la France, entre oîl et oc. L'Américain admire la découpe bleu de basalte de ses massifs, en venant honorer, au Puy, la mémoire de Lafayette, et Jules Romains, autre enfant du pays, y a planté quelques trétaux de son thêatre.

Le versant nord, aux récoltes maigres, prises entre un printemps toujours tardif et un hiver souvent précoce, aux voies d'accès difficiles, retiré, austère, fut longtemps une terre d'asile - huguenots, jadis, bravant les dragons du Roy, juifs, naguère, fuyant les sbires d'Himmler —, une sorte d'enclave méconnue, quelque part entre Lyon, Saint-Étienne et Valence.

Quand je m'y installai, le rude berceau familial prenait son essor. Les éléments qui avaient joué contre lui se tournaient en sa faveur. Village d'enfants, puis cure d'air pour citadins repus de fumée et de bruit, enfin centre pédagogique d'altitude, il était en train de devenir un site touristique privilégié.

Un Américain ayant déposé ses fils au collège privé, me rendit visite. En 1947, si j'ai bonne souvenance. Pour plus de précision, l'année où les États-Unis, ayant reconnu l'efficacité du B.C.G., menaient campagne, sur tout leur territoire, en faveur de ce procédé d'immunisation. Ce père précautionneux avait traversé l'Atlantique bien décidé à mettre ses garçons à l'abri de redoutables bacilles qui, sans nul doute, devaient pulluler dans une Europe convalescente. «En Amérique, me déclara-t-il, on vaccine maintenant contre la tuberculose. Connaissez-vous le Bi-Ci-Dji ?

- Vous pouvez prononcer le Bé-Cé-Gé, lui répondis-je, en souriant. Ce sont les initiales des médecins français qui l'on découvert et préconisé au début du siècle. Je l'emploie couramment, y compris dans ma famille. $\gg$ Et je désignai de la main deux de mes enfants qui jouaient devant la maison. Il m'examina d'un air quelque 
peu réprobateur, surpris de l'énormité de ce qu'il considérait comme une plaisanterie. Le Bi-Ci-Dji, produit en France ? Et allez donc !

À la décharge de cet authentique yankee, je rappellerai qu'à cette époque, nous en étions encore, et pour plusieurs années, aux «bons monnaie-matière »; que je fournissais à un industriel stéphanois du beurre pour acquérir «légalement » des lits métalliques destinés à monter une maison de repos, et que le spectacle de notre pays était plutôt celui de la faillite économique que de la médecine avancée.

Une langue ne vaut que par le peuple qui la parle et par l'idée que les autres peuples s'en font. L'oppression, les déportations, les compromissions semblaient avoir consommé, pour un voyageur non averti, la ruine de la France et de la culture française.

Cette image survécut longtemps à notre crise dans l'esprit de l'étranger, des Américains surtout. Peut-être se renforçait-elle du portrait avantageux que tout vainqueur est porté à se faire de lui-même.

Quelques années après cette conversation bécégiste, le collège privé, jusqu'alors installé dans des baraquements de fortune, inaugura son premier bâtiment en dur. La générosité américaine était pour une large part dans cette réalisation. Un ecclésiastique prononça une allocution au nom des donateurs. Bien que son français fut très honorable, il éprouva le besoin d'en excuser les imperfections en alléguant l'emploi de plus en plus répandu de la langue du Nouveau Monde. Sans aller jusqu'à proclamer que son expansion dans le reste du globe rendrait un jour la nôtre inutile, il révéla à l'auditoire qu'aux dernières informations - de la source desquelles on ne pouvait douter en raison des accointances célestes de l'orateur - la désignation de l'anglais comme langue officielle du Paradis était un fait acquis. Je ris le premier. Plus tard, me remémorant l'anecdote, je décelai, sous la boutade de cet homme de bien, la conviction latente d'une hégémonie linguistique.

\section{DÉPART INCERTAIN D'UNE CROISADE}

Quinze ans plus tard, cette conviction s'était confirmée au point que notre réaction contre la suprématie linguistique de nos anciens alliés parut à nombre d'entre eux puérile et ridicule.

Je revois une rencontre à Paris, alentour de 1965, en pleine offensive de «Clair-Dire » contre 1'《Anglo-saxonite épidémique» en France. Avec deux ou trois autres illuminés j'avais stigmatisé le đéfaitisme langagier du corps médical français devant un auditoire peu disposé à se laisser convaincre.

Quelques étrangers nous avaient honorés de leur présence, qui demeurèrent parmi nous pendant le vin d'honneur. Ainsi, fus-je pris, le verre de l'amitié à la main, entre un confrère et une consœur américains qui me tinrent à peu près ce langage : "Ainsi, selon vous, à tous les mots anglais on pourrait opposer un équivalent français? » J'opinai affirmativement. Le sourire de la dame s'effaça et le visage du monsieur prit un air de commisération. "Alors, traduisez-nous feed-back et stress !» 
Le Comité d'étude des termes techniques français et notre propre comité s'étaient attardés sur ces termes, dont le contexte est le plus souvent nécessaire pour en résoudre la polysémie. "En pratique, répondis-je, rétroaction et agression ${ }^{1} . \gg$ Tous deux se récrièrent. Feed-back et stress étaient des entités anglosaxonnes intangibles, inexprimables dans une autre langue, et définitivement consacrées!

Mais c'est parmi les médecins français, et non nécessairement des moindres, que se recrutèrent les anglolâtres les plus intraitables.

Revenons au début de l'année 1963. Février. La presse professionnelle publie le calendrier des congrès. Je lis : « $\mathrm{Au} \mathrm{XII}^{\mathrm{e}}$ congrès d'anesthésiologie... une cinquième discussion sera planifiée par le professeur agrégé Kern sur un sujet qui sera communiqué ultérieurement. »

Reprenant un vœu que j'avais récemment développé à l'adresse du corps médical ", j'écris le 28 février 1963, au $\mathrm{D}^{\mathrm{x}}$ Kern : "Une suggestion : n'appartiendrait-il pas à chaque discipline de mettre à l'ordre du jour de ses congrès un petit chapitre sur la terminologie. Les spécialistes présents versés dans la langue (il y en a toujours quelques-uns dans un groupe de médecins) pourraient aider à préciser le sens des mots nouveaux, à les corriger s'ils sont défectueux, et à en proposer - s'ils sont d'origine étrangère - des équivalents concis et colorés. Entre autres malentendus on éviterait ainsi ceux qu'engendrent la malfaçon ou l'imprécision des mots, des mots supports des idées et des objets, des mots instruments de travail. »

Kern, promoteur de l'anesthésiologie moderne en France dès la fin des hostilités, ce pionnier auquel, trois ans après sa mort ${ }^{3}$, les participants du Congrès mondial de Kyoto (août 1972) rendirent un unanime hommage, m'appelait son ami des bons et des mauvais jours.

L'an suivant, il me proposa d'entrer en relations avec son coéquipier des Cahiers d'anesthésiologie, le $\mathrm{D}^{\mathrm{r}}$ Jean Valletta qui, comme ce dernier me le rappelait récemment, "avait déjà éprouvé le besoin de mettre un peu d'ordre dans le langage utilisé à l'époque pour se mentir, en anesthésie comme ailleurs, certains auteurs faisant de la confusion des esprits leur tremplin ».

C'est ainsi que, le 5 juin 1964, assisté de plusieurs maîtres de l'anesthésie et de «mordus de la langue » (Bretagne, Guilmet, Huguenard, Kern, Lassner),

1. En répondant agression je n'avais fait que reprendre le vocable utilisé peu auparavant par le $\mathrm{D}^{\mathbf{r}}$ Selye lui-même, le père du stress, qui, lors d'un exposé à la télévision canadienne, avait choisi cet équivalent pour en expliciter le signifié (fait rapporté par le $D^{r}$ Jacques Boulay). On sait cependant les violentes passes d'armes qu'échangèrent partisans et adversaires de stress. Or, ce combat, qui semblait ne devoir jamais finir, déjà faiblit. Stress est en perte de vitesse. Sans doute le galvaudage qui en a été fait a-t-il terni son éclat. Sous la plupart des plumes françaises il avait pris les acceptions courantes de choc, jusqu'à s'appliquer, par exemple, à des contrariétés et à l'appréhension chez un enfant. Actuellement des articles entiers sont publiés, traitant du choc et de ses divers mécanismes - y compris la décharge hormonale dans l'organisme - où le terme anglais n'apparaît pas. Notons, au surplus, que choc y est écrit à la française, alors qu'il y a peu la graphie shock prévalait.

2. "L'anglo-saxonite épidémique ou Contre l'exercice illégal du langage médical français", l'Hôpital, janvier 1963.

3. E.R. Kern a raconté dans ses mémoires, Mes quatre vies, sa prodigieuse odyssée de combattant et d'anesthésiste (Paris, Librairie Arnette, 2, rue Casimir-Delavigne). 
Jean Valletta présida, dans un amphithéâtre de l'hôpital Cochin, le premier «Colloque médical sur la sémantique ».

J'avais, pour ma part, obtenu le concours d'un confrère non spécialiste, avec lequel je sympathisais dans la lutte contre le jargon médical : le $\mathrm{D}^{\mathrm{r}} \mathrm{O}^{\prime} F$ ollowell. Il aurait aujourd'hui cent un ans. Féru de latin et de grec, très pointilleux sur la correction langagière - il aurait d'ailleurs proscrit néologisme -, il s'évertuait, presque seul, depuis un demi-siècle à remettre notre vocabulaire sur la voie d'un classicisme rigoureux. C'était trop exiger ${ }^{4}$. Son éminent et respectable message passa sans l'atteindre au-dessus de l'auditoire estudiantin.

De son héroïque sagesse je retins surtout sa fermeté à l'encontre de ce qu'il considérait comme un des plus graves facteurs de corruption du français : son invasion par la terminologie anglo-saxonne.

"Dans le creux de la main d'un nouveau-né, mettez l'un de vos doigts... Aussitôt la main du bébé se ferme, manifestant ce qu'on appelle le reflexe de préhension. C'est trop simple évidemment; cela veut trop bien dire ce que cela veut dire. C'est pourquoi des médecins écrivent qu'ils ont constaté la présence d'un grasping normal!

Un chirurgien forme dans l'os une cavité puis il en abrase les bords... Aplanissement? Mais non! Vous apprenez qu'il fait de la «saucérisation ». De l'anglais saucer, soucoupe!»

Ces deux exemples - vogue des désinences en ing, francisation de verbes étrangers ayant déjà leurs correspondants dans notre langue - illustraient admirablement ce dont l'équipe Clair-Dire, noyau du Comité d'étude des termes médicaux français, faisait un des objectifs essentiels de sa campagne.

Cette offensive était sous presse. Elle éclata, huit jours plus tard, dans quatre grandes revues professionnelles sous la forme d'articles servant d'introduction à un travail original, la première liste des anglicismes médicaux et de leurs équivalents : cent trente termes ${ }^{5}$.

C'est donc à cette date, juin 1964, qu'une équipe de trois médecins — aidée d'un traducteur francophone, spécialisé en terminologie médicale et pharmaceutique ${ }^{6}$ - porta l'affaire devant le corps médical français.

4. Détail curieux. Quinze ans avant que le Comité d'étude des termes techniques français ne lançât une enquête sur le meilleur équivalent à donner à picturephone, il citait en le condamnant celui qui, retenu par l'I.B.M., vient d'entrer dans l'usage : «Comment qualifier le mot « vidéophone » pour nommer l'appareil qui permet, en téléphonant, de voir l'image de son correspondant!» Pour ce lettré, tout mâtiné était un monstre, et vidéophone ne trouvait pas plus grâce à ses yeux, que "cocktail lytique " ou " training autogène "!

5. Marcel Monnerot-Dumaine, «La traduction française des termes médicaux anglais », Presse médicale, 13 juin 1964 ; Jean-Charles Sournia, "Le franglais en médecine ", Supplément de la Semaine des hôpitaux, 14 juin 1964; Jean-Charles Sournia, "Les anglicismes médicaux », le Concours médical, 13 juin 1964; Daniel Eyraud, "La mare aux jargonautes », l'Hôpital, mai et juin 1964.

6. Henri van Hoof, directeur de l'Ecole des traducteurs de Bruxelles, traducteur près la Cour d'appel de Bruxelles. 


\section{JEUX-ENQUÊTES}

Sept mois à peine s'étaient écoulés que la phalange initiale donnait le jour à une association régie par la loi de 1901. Sous le parrainage de M. Alain Guillermou - auquel deux ans plus tôt je m'étais ouvert de mon projet - dans l'amphithéâtre Trousseau du vieil Hôtel-Dieu où des mâ̂tres enseignèrent dans une langue exemplaire, le Comité d'étude des termes médicaux français Clair-Dire reçut le baptême.

C'était le matin du 31 janvier 1965, un dimanche. Aux voix des quelques participants se mêlaient celles des cloches de Notre-Dame. Le professeur Maurice Lamy, le premier titulaire en France d'une chaire de génétique et seule notabilité présente, accepta courageusement de se compromettre dans l'aventure. Il devint, et il demeure notre président.

Ce jour-là, de l'initiative privée, naquit, en France, ce que le ministère des Affaircs culturelles au Québec avait déjà officiellement institué dans sa province ${ }^{\top}$, montrant à nos propres gouvernants, avec dix ans d'avance, la voie du dirigisme linguistique.

Rapidement les membres de Clair-Dire acquirent la conviction que rien de valable et de durable ne pourrait s'obtenir sans mobilisation de l'opinion. À cette époque nulle intervention des gens en place n'était ni décelable ni prévisible. Force était donc au nouveau comité d'asseoir son autorité sur un seul arbitrage, celui des usagers, en l'occurrence les médecins. Nous y consacrâmes l'année 1966.

L'intérêt porté par un grand laboratoire français ${ }^{8}$ au prestige de la France et de la langue française, donc à notre cause, nous permit de toucher, par le biais de deux jeux-enquêtes, près de quarante mille confrères. Le second, le plus important (vingt-cinq mille plis individuels), nous valut $8 \%$ de réponses, taux, parait-il, rarement atteint par les questionnaires. Nous avions porté aux débats quinze anglicismes suivis d'équivalents de notre choix : "Voulez-vous chercher avec nous? $\gg$

Le référendum fut formel. Sur deux mille réponses, $70 \%$ des correspondants rejetaient en bloc les anglicismes, $7 \%$ n'en toléraient qu'un seul, $8 \%$ en acceptaient seulement deux, $3 \frac{1}{2} \%$ en admettaient trois. Sur ce concert d'approbations tranchaient quelques notes d'une discordance violente, qui me rappelèrent un propos tiré par le $\mathrm{D}^{\mathrm{r}} \mathrm{O}^{\prime}$ Followell de sa longue pratique : «Les réactions des confrères ne sont pas toujours aimables quand on les reprend sur le langage. »L'année ne se passerait pas sans que, au cours d'un colloque que j'évoquerai plus bas, nous ne vérifiions la justesse de cette assertion.

Néanmoins, le référendum, par les préférences et les annotations de la grande majorité, nous apporta la preuve que le praticien était excédé par la gêne grandissante que lui suscitait, dans la mise à jour quotidienne de ses connaissances,

7. Comité d'étude des termes de médecine du Québec : président, $D^{r}$ Georges Desrosiers, secrétaire général, $D^{r}$ Jacques Boulay.

8. Les laboratoires Roussel : directeur délégué, M.G.A. Marchal. 
le flot des termes étrangers. Alors même que l'explosion des sciences rendait la matière médicale plus complexe, son vocabulaire, par une véritable aberration, allait en s'obscurcissant. D'autant que le grand chic de la plupart des m'as-tu-lu anglomanes était de ne fournir aucune traduction, bien qu'ils fussent censés écrire pour la multitude des médecins à la tâche.

Instruit et stimulé par le dépouillement du sondage, le comité Clair-Dire remettra sur le métier la liste d'anglicismes et l'étendra à 270 termes. L'appui de la même firme pharmaceutique lui permettra de donner à ce travail la présentation et la diffusion nécessaires. Sous la forme d'une élégante brochure, pourvue d'une introduction explicite, suivie de commentaires justificatifs, la Table d'anglicismes médicaux et de leurs équivalents sera distribuée aux médecins, dans le courant de l'année 1968, par les visiteurs du laboratoire ${ }^{9}$.

\section{L'ÉCHAUFFOURÉE DE VERSAILLES}

Mais restons à 1966, année charnière, année difficile. Les organisateurs du Colloque de Cochin avaient été des anesthésistes. C'est au sein d'un congrès de la même discipline que prit place, fastueux, le Colloque de Versailles. Une tradition se constituait. Pourquoi ?

$\mathrm{Au}$ lendemain de la guerre, l'anesthésie, en France, était figée dans une routine vieille de cent ans. Acte mineur, elle était abandonnée à des mains novices. Du jour au lendemain un étudiant du P.C.N. se trouvait armé du redoutable masque d'Ombredane sous la conduite empirique d'une bonne sœur. Anglais et Américains étaient à cet égard nos devanciers, et il fallait, pour répandre l'anesthésie moderne chez nous, transposer leur vocabulaire dans notre langue. La recherche d'équivalents adéquats s'imposait plus que dans toute autre spécialité.

Nous voici à Versailles, le 5 juin 1966. Le président Evrard, anesthésiste belge, prévoit une séance houleuse, comme il ressort des prises de positions publiées, en avant-programme, dans les Annales de l'anesthésiologie française ${ }^{10}$. Son rédacteur en chef, ayant titré sa communication «Défense et illustration du franglais », développe sa thèse. À l'ère du langage-machine (sic) un seul terme doit prévaloir dans l'univers médical. Ce terme doit être celui que le chercheur a pris dans sa langue maternelle, quand bien même il serait en soi un terme médiocre. Ainsi, d'après ce confrère, le vocabulaire médical du monde entier était réduit au champ sémantique du chercheur et soumis à son inspiration. Mots courants, banals, cocasses, voire argotiques, assonances et télescopages, se trouvaient haussés au rang d'expressions scientifiques universelles. Compte tenu de l'énorme avance américaine d'alors dans le domaine de la recherche, une pareille attitude garantissait la suprématie de l'anglais.

Le goût de l'orateur l'ayant porté sur sludge, la crasse des garagistes yankees fut prise par cet anesthésiste, titré et chef d'école, comme modèle de « motmachine $»$.

9. En 1970 , un additif de 50 termes portera le total à 320 .

10. Annales de l'anesthésiologie française, $7^{\circ}$ année, $\mathrm{n}^{\circ} 2$. 
Je le cite : "Quelques exemples illustrent cette notion : il est impossible d'inscrire sur une carte perforée le phénomène qui consiste en... (suivait une définition de six lignes du genre encyclopédique). Pour les physiologistes, biologistes et anesthésistes du monde entier ce phénomène s'appelle sludge ${ }^{11}$ ! »

Des médecins avaient déjà adressé à Clair-Dire des protestations, dont celle-ci, émanant d'un grand patron parisien : «Ce n'était pas assez des postes à essence pour introduire sludge chez nous. Certains confrères s'en emparent!» Or, la solution avait été donnée par un anesthésiologiste six mois avant : «Le terme anglo-saxon nous paraît insuffisamment précis et descriptif pour être introduit dans notre langage médical. Celui d'agrégat nous paraît donner la meilleure description đu phénomène ${ }^{12}$. »

Entre-temps sludge avait accouché de deux avortons sludage (sic) et, évidemment, slugging; et on étudiait des produits anti-sludge. Mais, entre-temps aussi, d'autres pays, banissant sludge et sa lignée, - Allemands, Italiens, Espagnols avaient adapté à leurs normes linguistiques l'ancêtre latin aggregatio. Mieux encore aggregation était apparu dans les écrits anglo-saxons.

Le président Evrard estima opportun d'en finir avec cette controverse en faisant obscrver que, les Allemands disant agregation et les Anglais aggregation, il serait comique de trouver dans un dictionnaire quadrilingue, en regard de ces termes, le français « sludge $»$ !

Les applaudissements avaient à peine salué ce trait spirituel qu'un coéquipier du défenseur du franglais, relança la balle en exhumant une communication vieille de quarante ans où un physiologiste étranger, pour décrire un phénomène approchant, avait utilisé aggregation. L'argument, purement historique, venait à l'appui de la déclaration que ce maître, en début de séance, avait introduite par la précaution oratoire que voici : «Pour que les mots ne dépassent pas ma pensée, je vous demande que je vous lise le texte de cette communication.»

Après quoi nous avions entendu : «Il ne s'agit pas de savoir si un équivalent existe dans notre langue pour remplacer un terme étranger. Déguiser une pensée étrangère avec un mot français... est tout simplement malhonnête. » Dans sa péroraison il avait évoqué Déroulède, puis, feignant l'indignation, il avait cité un article américain où le signataire, faisant état de la réaction qui s'amorçait chez nous contre le franglais ${ }^{13}$, mettait un point final à ses sarcasmes en employant une locution française «C'est la vie ». «En le lisant j'ai rougi », expliqua l'argumenteur; bouffée vaso-motrice qui, évidemment, chez un spécialiste de l'hibernation, exprimait le fond de la honte. Mais, au fait, avait-il rougi ou ressenti un flush?

Il est à croire que chez certains enseignants, une affirmation une fois lancée s'incorpore dans leur théorie pour y prendre l'aspect d'une vérité première.

11. Plaquette commémorative de l'Association internationale des anesthésistes-réanimateurs de langue française : président, $D^{r}$ Camille Evrard; secrétaire général, $D^{r}$ Jacques Raickman.

12. J.C. Bernard, l'Hôpital, décembre 1963.

13. Et non, je le répète, contre l'enseignement de la langue anglaise, ce qui est tout autre chose. 
Le temps coule. Tout paraît changé. Cependant l'affirmation reste immuable. Le 15 décembre 1972, devant les mêmes participants, l'orateur reprendra textuellement ses propos et, appliquant derechef à notre recherche d'équivalents le qualificatif de malhonnête, confirmera : «Le langage scientifique est à celui qui le trouve. » Ce à quoi, le président de Clair-Dire, promu depuis président de la Commission du langage médical auprès du ministère de la Santé publique, répondra dignement : "Bien sûr que non! Ce qui appartient au savant c'est la trouvaille et non le mot qui la désigne, qui peut être vague, incongru ou argotique. »

Sur ce point névralgique du Colloque de Versailles, un auditeur neutre aurait conclu : «Laissons trancher l'usage. »

Eh bien! C'est chose faite. En effet, un équivalent a gagné : 1) lorsque le dictionnaire l'a enregistré. Or, ouvrez le Nouveau Littré médical, le Manuila. Sludge y est mentionné, mais il renvoie à agrégat et c'est à agrégat qu'on trouve la description du phénomène ; 2) lorsque la publicité médicale l'a adopté. Consultez nos revues médicales, les laboratoires ont délaissé le terme anglo-saxon, et non seulement ils usent d'agrégat, d'agrégation, mais encore ils leur ont donné les dérivés dont ils avaient besoin : ils proposent aux médecins des produits antiagrégants pour lutter contre l'agrégativité ${ }^{14}$.

\section{L'ANGLOMÉGALIE}

De nouveau rétrogradons dans le temps jusqu'à cette époque où les coéquipiers de Clair-Dire faisaient figure d'une quadrette d'illuminés, dont le clan claironnant des anglomanes se riait, que les médecins haut placés regardaient faire avec un recul prudent, et à laquelle les confrères informés témoignaient cette sympathie mêlée d'apitoiement que l'on réserve aux défenseurs chevaleresques des causes perdues d'avance.

À partir de 1960 environ, le néo-jargon était devenu dans notre littérature médicale plus qu'une mode, une obligation. Rares étaient les articles, éditoriaux, comptes rendus de tables rondes, analyses, extraits qui ne s'assortissaient d'une floraison de mots chocs, de mots «stressants» aurait-il fallu dire. Ces mots semblaient disposés intentionnellement dans le texte, qui pouvait être d'une réelle valeur scientifique, comme pour accrocher l'œil du lecteur, en fait le déroutant, le lassant ou l'exaspérant.

«Comme on se sent petit devant de tels mots», ironisait un médecin de province, en commentaire à l'un de nos jeux-enquêtes. Le fin du fin consistait à étoiler son discours d'un mélange judicieusement dosé de néologismes (parfois à soi), de vocables rares ou déviés (genre critique d'art), de pathos psychanalytique et surtout d'anglicismes. À propos de ceux-ci, leur nouveauté attestait la frâ̂cheur d'une information recueillie soit dans un colloque international où avait dominé l'anglais, soit dans des publications américaines. Ces m'as-tu-lu savaient d'ailleurs

14. Au Colloque sur la norme, tenu le 3 mai 1968 par le Conseil international, le professeur Etiemble ramena les congressistes sur terre en lançant d'une voix véhémente : "Nous sommes soumis au normatif de la publicité !...» Que cet exemple soit pour lui un hommage et lui apporte l'assurance que les retombées de sa bombe de 1964, « Parlez-vous franglais $"$, ne sont point inactives. 
comment mâter l'irritation du lecteur. Que si un praticien rural ou de quartier s'était avisé d'adresser une lettre ouverte au comité de rédaction, sa lettre aurait pu être reproduite, mais suivie d'une mise au point si bien «gratinée » que l'affaire aurait tourné à la confusion du fâcheux. Ainsi, pratiquement incontestée, l'anglofolie atteignit des sommets.

On vit s'étaler des anglicismes non seulement en cours de texte mais en titre. En voici une brochette, prélevée dans ma «franglothèque», où, par discrétion, la partie en français a été démarquée : FEED-BACK du sorcier; HIT PARADE de la névralgie; Paludisme FOR EVER; FLASH BACK sur le colloque de Villejuif, etc.

Le clou fut, à mon sens, un article intitulé CHALLENGE of LIFE, dans lequel l'auteur, par des arguments spéciaux émis sur le mode péremptoire, s'en prenait aux lecteurs qui avaient trouvé de mauvais goût cette appellation choisie par un grand laboratoire français pour célébrer, dans une réception-débat somptuaire, le soixante-quinzième anniversaire de sa fondation.

Les protestataires ayant été relégués au rang de «preux chevaliers d'un tournoi anachronique », et une évocation du vieux français chalange ayant attesté les connaissances linguistiques de l'argumenteur, ce dernier pérorait « Reste-t-il quelque chose de cet ancêtre fielleux dans l'actuel «challenge »?

Ce qui fait la nocivité de pareils exemples et de pareille dialectique, c'est que la plume des médecins - si individualistes pourtant - est curieusement moutonnière. Tout vocable neuf dans sa forme (néologisme, emprunt), altéré dans son signifié (glissement de sens indigène ${ }^{15}$, faux-ami ${ }^{16}$, insolite ou fanfaron ${ }^{17}$, se propage en quelques mois d'un auteur à l'autre, à la façon d'une maladie contagieuse.

Les autcurs attitrés de tel hebdomadaire professionnel se «refilent 》 des locutions entières, parfois en les écorchant, jamais en les traduisant. On écrit happy end pour heureux dénouement (d'un cas difficile); no man's land pour marge d'incertitude ou part d'inconnu (diagnostique, thérapeutique); et quand on veut clore en beauté une énumération de symptômes, le dernier mais non le moindre manquant de prestance, on s'enfle d'un the last but not the least 18 !

Des anglicismes viennent prendre la place d'expressions françaises antérieures et consacrées. Le roulement mésodiastolique, décrit par Durozier et Potain il y a un siècle, devient le mid diastolic rumble; le pontage, inventé en France par Kunlin, le by-pass ${ }^{19}$; l'écart type, la standard deviation ou la déviation standard ${ }^{20}$.

Cette fausse honte à borner son vocabulaire au seul français ne va pas d'ailleurs sans cuirs irrévérencieux envers la langue anglaise. Que penser de «kinking » de la crosse aortique, coudure, coque, écrit « King King 》 (réminiscence

15. Récupérer pour prenđre, vocation pour rôle, mutation pour évolution.

16. Réhabilitation pour rééducation, opportunité pour occasion, systémique pour général.

17. Exhaustif pour complet ou total, extrapoler pour transposer ou déduire, paramètre pour facteur ou constante.

18. Prof. Yves Bouvrain, Leçon inaugurale, 24 mars 1966.

19. Dr Gerson, Lex et Scientia, janvier-mars 1969.

20. Prof. Maurice Lamy, Table d'anglicismes, 1968. 
d'un film de terreur ?) ; des pluriels flushs, boxs, rashs (les rashs aux pénicillines) ? Éruption ferait banal. Érythème plus savant manque de griffe. Alors, revenez au vieux français « rache », père du mot anglais, et ajoutez-y sans crainte un $s$ au pluriel ${ }^{21}$ !

\section{LA RÉALITÉ D'AUJOURD'HUI EST FAITE DE L'UTOPIE D'HIER 22}

Mais pourquoi irai-je plus avant dans le procès de l'anglomanie, en ce début de l'année 1973, alors qu'il semble présenter, depuis peu, un intérêt plus récapitulatif qu'actuel, la cause étant officiellement jugée.

Le pouvoir a compris la valeur de l'enjeu et sans doute l'a-t-il incorporé à son programme de l'Europe de demain. Le journal officiel vient de publier une liste de proscrits et de donner leurs équivalents, entérinant ainsi les recommandations des commissions ministérielles, elles-mêmes inspirées pour l'essentiel des travaux de groupements descendus dans l'arène depuis plusieurs lustres. Je dis descendre dans l'arène et s'y mesurer au corps à corps avec les intrus, car il s'agissait bel et bien d'une bataille dont les combattants étaient des francs-tireurs français et francophones, et parmi ceux-ci, aux postes les plus exposés, canadiens.

Toutefois, l'étude comparative des publications médicales au cours de ces dernières années démontre que, avant même cette sanction gouvernementale, notre campagne avait porté des fruits. Des chefs d'école avaient pris conscience, comme nous les y avions conviés, de ce que leur enseignement gagnerait en clarté et en efficacité à l'emploi de nos équivalents.

J'ai colligé, au cours des deux dernières années, des articles qui l'attestent et où pourtant la tentation de glisser l'anglicisme était forte.

Ainsi, sous le titre Cinquante ans de cardiologie, le premier des maîtres lyonnais dans cette discipline emploie exclusivement stimulateur et pontage ; tandis que l'auteur parisien du traité le plus autorisé de cardio-angiologie ignore by-pass et patch pour décrire le pontage veineux aortico-coronarien et la pose d'une pièce sur un vaisseau. L'hémodialyse à domicile est rapportée par un néphrologue sans que shunt ou by-pass apparaissent, mais seulement court-circuit, et les petits reins artificiels sont pourvus de plaques jetables et non disposables.

Enfin, dans l'immense domaine du langage médical en général, un événement d'une portée considérable s'est produit. Ce qui était réputé impossible est devenu réalité. Le hallier de l'idiome médical a été inventorié, sa proliférante végétation élaguée et mise en ordre.

Pour mesurer le chemin parcouru il faut se reporter à la déclaration faite, voici bientôt quinze ans, par un de nos plus grands écrivains, auquel l'exercice de la chirurgie de l'avant inspira ses premières cuuvres, Georges Duhamel : «Il existe à l'Académie de médecine une Commission du dictionnaire qui existait déjà

21. "On dirait que les milieux français cultivés sont incapables de se servir du fonds populaire pour se forger des termes nouveaux. "Aurélien Sauvageot, Portrait du vocabulaire parlé, Paris, Larousse, 1964, p. 233.

22. Paraphrase d'une pensée d'André Gide. 
en 1937 quand je devins académicien. Elle est rarement convoquée pour la raison que l'évolution du langage médical est telle que le dictionnaire serait périmé avant d'être publié ${ }^{23}$. 》

Or, tandis que notre illustre confrère exprimait le désarroi de nos plus hautes institutions devant l'expansion formidable du vocabulaire médical, le docteur Alexandre Manuila se lançait dans la composition d'un dictionnaire de médecine et de biologie et, communiquant sa foi à une élite de collaborateurs, construisait un édifice quatre fois plus vaste que le Littré médical et que le Dictionnaire général de l'Académie française ${ }^{24}$.

Chaque terme y fut affecté d'un sens précis, l'anarchie de la synonymie fut résolue, la communication entre médecins fut rétablie. Le praticien, arrêté dans sa lecture par un anglicisme, l'y trouve dans l'ordre alphabétique, mais, le plus souvent, avec un renvoi à l'équivalent français qui, lui, en donne la définition. Doping renvoie à dopage, dumping syndrome à syndrome de chasse, wrapping à enrobage, et on lit en regard de l'anglicisme : «anglicisme utilisé à tort mais couramment dans les textes français comme synonyme de ... ».

Manuila en main, le prôneur de franglais acceptera-t-il de revenir sur son erreur et de concéder au rédacteur en chef de l'Encyclopédie médico-chirurgicale, le $\mathrm{D}^{r}$ Olivier Marty, que « la confusion [du langage médical] est encore augmentée par la propension des médecins français à se précipiter sur les termes anglosaxons pour les utiliser, en général dans un sens vague et imprécis, souvent dans une acception différente de celle utilisée Outre-Manche ou Outre-Atlantique ${ }^{25}{ }$.

Nous ne pouvons pas ne pas saluer au passage cet autre monument publié l'année écoulée, dans lequel, à la satisfaction de la difficulté sémantique, graphique ou syntaxique résolue, s'ajoute l'agrément de la découverte dans un texte disposé avec une attrayante clarté : l'Encyclopédie du bon français dans l'usage contemporain ${ }^{26}$. Dans sa préface, le président du comité de rédaction, M. Fernand Keller, prend position : "Nous avons aussi consacré des articles à des mots étrangers qui sont passés tels quels dans un vocabulaire technique français, mais que leur emploi répété a rendu familiers à nos oreilles. Nous avons tenté de nous rendre compte si leur usage était légitime ou non et si, dans la négative, il était possible de proposer un équivalent satisfaisant. » Ici l'auteur rend un hommage particulier au Comité d'étude des termes techniques français, mais le lecteur qui consulte souvent le Dupré peut y trouver mention de Clair-Dire dont les préférences sont ratifiées.

Aux côtés du Manuila et de ses 150000 termes, se situent les glossaires médicaux dont le contenu va de quelques centaines à plusieurs milliers de mots.

23. Le Dauphiné libéré, août 1959.

24. A. et L. Manuila, M. Nicole et H. Lambert, Dictionnaire français de médecine et de biologie, 3 vol., Paris, Masson, 1970-1973.

25. Olivier Marty, "Instantanés médicaux ».

26. Trois tomes, Paris, Editions de Trévise, 1973, avec la collaboration de Jean Batany. 
En 1970, notre Comité d'étude, avec l'appui du Conseil international de la langue française, publia un dictionnaire spécialisé qui, par la matière choisie, constituait une gageure.

Dans un ouvrage riche de 2500 expressions, le docteur Pierre Marchais, s'attaquant au vocabulaire médical le plus malaisé à circonscrire, celui de la psychiatrie, démontrait de façon exemplaire que n'importe quelle spécialité scientifique pouvait faire l'objet d'un classement terminologique, et être rendue intelligible à qui voudrait prendre la peine d'en forcer la barrière des mots ${ }^{27}$.

Pour revenir sur le cas particulier des xénismes, lorsque l'un d'eux apparaît dans le lexique, le lecteur est renvoyé à son équivalent : behaviour therapy, border line, double-blind test, dreamy state, happening, insight, transvestism, sentencing, qui renvoient respectivement à comportement (thérapie du), cas limite ou état frontière, méthode à double insu, état de rêve, impromptu, intéroception, travestisme, jugement.

D'une façon plus systématique, une série de dictionnaires spécialisés viendra encore élargir la somme du grand Dictionnaire de médecine et de biologie, en recensant le vocabulaire de certaines branches médicales ou en donnant l'acception particulière que ces branches attribuent à un terme du fonds médical commun.

Le premier d'entre eux, intentionnellement présenté en même temps que le troisième tome du grand Dictionnaire de médecine et de biologie, confirme cette opportunité. Y sont traités 900 expressions, locutions ou sigles, dont tout Français bien portant ou malade, en activité ou à la retraite, peut avoir besoin de connaître la signification médico-socio-administrative. Il s'agit du Dictionnaire des assurances sociales, publié avec le concours de notre Comité d'étude.

En exemple. Le premier mot du lexique est abattement. Dans le grand dictionnaire abattement désigne une «diminution marquée de la vitalité » et renvoie à dépression. Par contre, le réalisateur du Dictionnaire des assurances sociales, le professeur Jean-Charles Sournia, médecin-conseil national, le définit ainsi : « Déduction faite sur une somme à payer (cotisation, indemnité, rente) ${ }^{28}$ ».

En vain, dans ce classement lexical médico-socio-administratif, on chercherait nursery, home d'enfants, birth-control, check up, mais on y trouvera crèche et pouponnière, maison d'enfants, bilan de santé, régulation des naissances.

27. Pierre Marchais, Glossaire de psychiatrie, Paris, Masson.

28. Le Comité d'étude des termes médicaux du Québec, présidé par le $\mathrm{D}^{\mathrm{r}}$ Georges Desrosiers, animé par son secrétaire général, le $\mathrm{D}^{\mathrm{r}}$ Jacques Boulay, avait, avant nous, été confronté avec ces difficultés que rendait aiguës l'aménagement à l'américaine des établissements hospitaliers canadiens. C'est ainsi que, sous un volume il est vrai beaucoup plus réduit, mais donnant en revanche les canadianismes fautifs, il avait publié un Vocabulaire des assurances sociales et des assemblées délibérantes, précédé d'un Glossaire des termes médico-hospitaliers et suivi d'un Glossaire des termes pharmaceutiques; brochures échelonnées de 1966 à 1969.

Tous ceux qui ont suivi les efforts des deux comités, de part et d'autre de l'Atlantique, savent à quel point - avant même la rencontre de leurs délégations à la biennale de Namur (1965), de Québec (1967), de Liège (1969) - leur coopération fut assidue. La Table d'anglicismes et de leurs équivalents, de 1968, a été valorisée par leur concertation. Un même souci d'efficacité leur inspire, au Colloque de Liège sur le langage médical, le texte d'une Charte de solidarité lexicale destinée à obvier à un récent facteur de confusion né du succès de notre commune entreprise, celui de la concurrence des équivalents. L'allocution d'un des délégués de Clair-Dire s'intitulait, le 10 octobre 1969 : Pour un français médical unitif. 


\section{PERSÉVÉRER ET ENTREPRENDRE}

J'ai écrit plus haut que la cause de l'anglomanie était officiellement jugée.

Ceci appelle quelque modération, car le vocabulaire s'accroît en proportion des sciences - voire des échanges culturels et commerciaux - et chaque notion ou découverte nouvelle peut exiger un signifiant nouveau.

D'autre part, l'usage a des caprices qui peuvent tenir en échec le dirigisme le plus sage. Enfin, dans le secteur de la médecine, existe une tradition d'indépendance où l'aversion pour toute contrainte entretient l'indiscipline, d'ailleurs souvent confondue par les intéressés avec la liberté de jugement ou la force de caractère.

Les groupements dont la saine évolution de la langue française est la raison d'être doivent donc se maintenir sur la brèche. Sans relâche il leur faut explorer le champ de l'expression écrite et parlée, en épingler les vocables, en saisir les tours nouveaux. Il leur faut les peser sans parti-pris de conservatisme, ne jamais les rejeter d'office sous le motif qu'ils ne figurent pas encore au dictionnaire, examiner objectivement si leur singularité ne répond pas à une commodité, à un enrichissement licites, entendons adaptables au génie de la langue. Un accommodement raisonné et raisonnable vaut mieux qu'une position dogmatique.

Ceci, non sans happer le nouveau venu au vol et en débattre sans délai, pour le gagner de vitesse s'il est condamnable et lui barrer la route par un équivalent approprié. Toutefois, ce serait un leurre que de borner son effort au criblage et à la transposition des emprunts.

Le français doit se regénérer par le dedans. Il est vital qu'il forge des mots comme cela a été dit excellemment au Colloque sur la créativité ${ }^{29}$ et comme le Comité d'étude des termes techniques français en fait la démonstration depuis près de vingt ans ${ }^{30}$.

Pour se régénérer il faut que le français s'ébroue, qu'il secoue le harnais conventionnel sous lequel il perd le souffle. Lettrés et grammairiens ne voudront-ils pas reconnaître que le recours aux composés savants n'est en fait qu'une facilité et que la systématisation du procédé compromet la vigueur de la langue ${ }^{31}$.

Accepteront-ils, leur savoir mis au service de l'efficacité, de se placer dans les rangs des artisans de la langue dont la seule ambition est de la rendre aisée, maniable, expressive, plaisante, et, prenant le contre-pied d'une manière dont l'ésotérisme médical représente un extrême, d'aider à l'enrichir en préservant son naturel et sa saveur.

La consultation de cet interlocuteur volontiers rétif qu'est le médecin nous a montré la meilleure façon de nous y prendre pour emporter son adhésion et, en somme, nous a conduits à ériger en principe ce qui aurait dû, dès l'abord, s'imposer à nous comme une évidence.

Légiférer, argumenter, chapitrer, vitupérer, loin de le convertir souvent l'indisposent. Sans doute a-t-il fallu le convaincre de la légitimité de notre action :

29. Conseil international de la langue française, Colloque sur la néologie lexicale dans la francophonie, mai 1971.

30. Essai d'orientation de la terminologie, Paris, Hermann, 1972.

31. Persuadons-nous, au surplus, qu'à tout prendre - et à tout apprendre - un étudiant d'aujourd'hui préférera l'original anglais à un amalgame grec controuvé pour la circonstance. 
la défense de la communication entre gens d'un même métier. Ce n'est pas assez. Il faut aussi lui plaire, et pour y parvenir, nos moyens les plus sûrs sont moins nos connaissances sémantiques - en admettant que nous en possédions assez pour en faire étalage - que notre bon vouloir désintéressé, notre sens des réalités, notre capacité d'invention et notre aptitude à faire surgir une image. C'est dans cette voie que nous poursuivrons notre effort.

D'autant que nous y sommes encouragés par un revirement partiel et récent, mais manifeste, en faveur des créations lexicales spontanées, évocatrices, agrémentées souvent d'un grain de fantaisie; en faveur aussi du style limpide et fleuri des grands cliniciens du début du siècle. Alors le choix et l'agencement des mots ne procédait pas du désir de paraître, mais de leur application à dispenser un enseignement accessible à tous; alors les transcripteurs des publications étrangères n'étaient pas frappés d'inhibition devant le flux terminologique déferlant d'Outre-Atlantique.

Le vent tourne. L'époque où la médecine française précellait dans le monde n'est plus seulement évoqué dans un contexte historique avec la nostalgie des choses surannées; la forme même d'expression dont elle nous a laissé l'héritage est citée en exemple aux auteurs actuels par des comités de rédaction. Je ne m'attarderai pas à reproduire une sélection des termes imagés nouvellement apparus qui, autrement mieux que xénismes, hexagonismes et grécismes, désignent et décrivent à la fois des découvertes modernes. Mais j'ai plaisir à mentionner une rubrique, publiée en cinq parties ${ }^{32}$, où une centaine de clichés et croquis commentés rappellent à la fois la charge et la valeur d'information d'une simple image.

Pour terminer j'en reviendrai à la vie de notre Comité. Ces pages ont survolé son passé. Ma conclusion annoncera ses projets pour l'année en cours. Ce sont, outre l'incessante prospection du vocabulaire nouveau, deux réalisations, au demeurant complémentaires. En premier, un ouvrage consacré au langage médical moderne, où toutes les expressions françaises et étrangères qui ont été discutées par le Comité seront étudiées et commentées. Dans un second temps, une publication à grand tirage diffusera parmi les médecins et tous ceux qui, de par leur activité emploient des termes médicaux, un condensé, mis à jour, des anglicismes médicaux et de leurs équivalents.

Puissent ceux qui les consulteront ne point se méprendre sur les mobiles qui les auront inspirés, et puisse la majorité de ceux qui font la langue les accueillir avec faveur, pour le plus grand bien des lecteurs qui veulent comprendre et pour celui des auteurs qui, à trois siècles de distance, se demandent comme La Bruyère « Est-ce un si grand mal d'être entendu quand on parle?».

DaNiel Eyraud

32. Guy Naudin, Max Potter et François Mignon, "Le chant du signe ", le Concours médical, 1969-1971. 